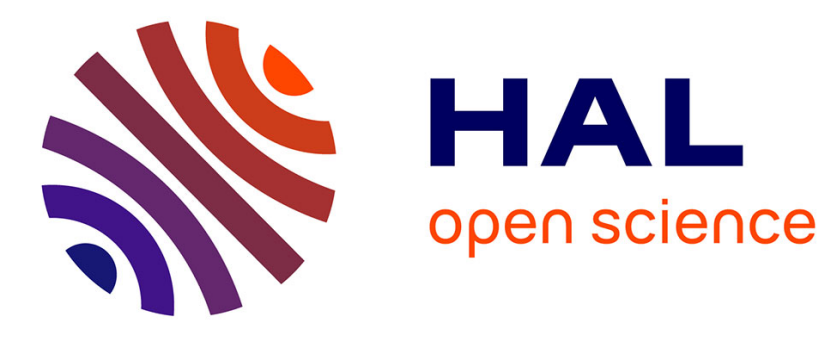

\title{
Dual gas and oil dispersions in water: production and stability of foamulsion
}

\author{
Anniina Salonen, Romain Lhermerout, Emmanuelle Rio, Dominique \\ Langevin, Arnaud Saint-Jalmes
}

\section{To cite this version:}

Anniina Salonen, Romain Lhermerout, Emmanuelle Rio, Dominique Langevin, Arnaud Saint-Jalmes. Dual gas and oil dispersions in water: production and stability of foamulsion. Soft Matter, 2012, 8, pp.699. 10.1039/c1sm06537h . hal-00974346

\section{HAL Id: hal-00974346 https://hal.science/hal-00974346}

Submitted on 7 Apr 2014

HAL is a multi-disciplinary open access archive for the deposit and dissemination of scientific research documents, whether they are published or not. The documents may come from teaching and research institutions in France or abroad, or from public or private research centers.
L'archive ouverte pluridisciplinaire HAL, est destinée au dépôt et à la diffusion de documents scientifiques de niveau recherche, publiés ou non, émanant des établissements d'enseignement et de recherche français ou étrangers, des laboratoires publics ou privés. 


\title{
Soft Matter
}

\section{Dual gas and oil dispersions in water: production and stability of foamulsion}

\author{
Anniina Salonen, ${ }^{* a}$ Romain Lhermerout, ${ }^{a}$ Emmanuelle Rio, ${ }^{a}$ Dominique Langevin ${ }^{a}$ and Arnaud Saint-Jalmes ${ }^{b}$
}

Received 10th August 2011, Accepted 28th September 2011

DOI: $10.1039 / \mathrm{c} 1 \mathrm{sm} 06537 \mathrm{~h}$

\begin{abstract}
In this study we have investigated mixtures of oil droplets and gas bubbles and show that the oil can have two very different roles, either suppressing foaming or stabilising the foam. We have foamed emulsions made from two different oils (rapeseed and dodecane). For both oils the requirement for the creation of foamulsions is the presence of surfactant above a certain critical threshold, independent of the concentration of oil present. Although the foamability is comparable, the stability of the foamed emulsions is very different for the two oils studied. Varying a few simple parameters gives access to a wide range of behaviours, indeed three different stability regimes are observed: a regime with rapid collapse (within a few minutes), a regime where the oil has no impact, and a regime of high stability. This last regime occurs at high oil fraction in the emulsion, and the strong slowing down of ageing processes is due to the confinement of packed oil droplets between bubbles. We thus show that a simple system consisting of surfactant, water, oil and gas is very versatile and can be controlled by choosing the appropriate physical chemical parameters.
\end{abstract}

\section{Introduction}

Aqueous foams and (oil in water) emulsions are both dispersions of water insoluble fluids (respectively, gas and oil) in water and are inherently unstable in time. ${ }^{1,2}$ Their widespread presence in our everyday lives has resulted in significant research activity investigating the reasons behind their limited stability. In order to create stable foams or emulsions, a first requirement is that stabilizing agents - such as surfactants - have to adsorb at the water-air and the water-oil interfaces, stabilising the dispersed bubbles or droplets against coalescence (fusion of two bubbles/ drops). However, the surfactants cannot generally fully prevent ageing of foams and emulsions with time through gravitational foam drainage or emulsion creaming, as well as through coarsening (or Ostwald ripening), i.e. gas or oil transfer between bubbles/drops due to capillary pressure differences. These two processes, together with coalescence, lead to a time-dependent destabilisation of the dispersions.

One of the current challenges is the creation of very stable foams, which means studies on the physics of the different mechanisms of ageing. ${ }^{3-5}$ Other research activities deal with the optimisation of the chemical formulation of foams, in order to reduce ageing as much as possible. A first approach consists of replacing surfactants by other stabilizers, ${ }^{6,7}$ such as proteins, or short polymers, with which ageing is slower than with surfactant foams or emulsions. A more drastic option is to replace surfactants by partially hydrophobic solid particles, which can create

${ }^{a}$ Laboratoire de Physique de Solides, CNRS-UMR 8502 Université Paris Sud, 91405 Orsay Cedex, France. E-mail: anniina.salonen@u-psud.fr

${ }^{b}$ Institut de Physique, CNRS-UMR 6251 Université Rennes 1, 35042 Rennes Cedex, France an incompressible armour around the bubbles, ${ }^{8-10}$ as in "Pickering emulsions". ${ }^{11,12}$ Though adsorbed solid particles can prevent coarsening and coalescence, ${ }^{9}$ drainage effects are still present in such foams. Efficient control of drainage has been achieved by doping the continuous phase with colloidal particles, self-assembled supramolecular structures, or polymers to create gelled and/or jammed structures within the network of liquid channels ("Plateau borders") containing the foam fluid. ${ }^{13-17}$ Nevertheless, in most cases, ageing is slowed down, but never fully suppressed.

The research, not only to increase and control stability, but also to add advanced functionalities to the systems has led to studies of more complex systems, such as multiple emulsions ${ }^{\mathbf{1 8 , 1 9}}$ or mixtures of droplets and bubbles. ${ }^{20}$ In particular, mixtures of bubbles and droplets are encountered in many cosmetic and food products (such as whipped cream) or in oil recovery processes. Depending on the applications, foaming can be either a desired (to improve the emulsion texture or sensory aspects, for instance) or an unwanted side effect. Mixtures of droplets and bubbles inside an aqueous matrix could be either considered as a foamed-or "aerated"-emulsion, or as an aqueous foam whose interstitial fluid is doped with oil droplets. In the most stable systems, the oil droplets are generally crystallised (at least partially), ${ }^{21}$ and act as solid particles. Although many studies on food grade foamed emulsions have been performed, ${ }^{22,23}$ fewer studies exist on simpler systems. In a pioneering work, Koczo et al. showed that bubbles and fluid oil droplets can coexist without destabilization of the foam. ${ }^{24}$ However, this study only dealt with relatively low concentrations of oil droplets in the foaming fluid (dilute emulsions). More recently, the presence of emulsion droplets has been shown to offer the control of the 
foamability using UV-light in the presence of light sensitive polymers. ${ }^{25}$

Introducing fluid oil drops inside a foam is the classical approach to suppress the foaming of a solution and to destabilize existing foams. ${ }^{26,27}$ Oil globules could act either as fast antifoams (emulsion droplets enter the air/water interface in the films and subsequently break them) or as slow antifoams (due to capillary suction, the emulsion droplets are chased out of the films into the Plateau borders where they enter the air-water interface once the borders have shrunk due to drainage, after which the foam collapses). ${ }^{26}$

However, all oils are not necessarily antifoaming systems. Whether an oil globule acts as antifoam or not depends in particular on the interfacial tensions between the three phases (gas, water, oil); one generally introduces various coefficients (spreading $S$, entering $E$ and bridging $B$ ) to describe the antifoam potential of an oil. The situation is complex and not yet fully understood, these coefficients have to be used along with an entry barrier coefficient, which appears the best measure of antifoam activity i.e. how easily the droplets can enter the air/water interface. ${ }^{26}$

In this article, we have investigated how large volumes of controlled dual oil-gas dispersions (called foamulsions, in the following) can be produced and what are the key parameters controlling their creation and their ageing. Our strategy is to foam a preformed model emulsion, made from two different oils (with different affinities toward water, in order to possibly control the antifoam action), varying the oil content and the concentration of surfactant. In particular, we have looked for possible experimental conditions leading to the most stable systems, where the droplets and bubbles synergistically stabilise the structure. The results show that oil droplets can be used for both foam destabilisation or to reach outstandingly long foam lifetimes.

\section{Experimental section}

\subsection{Materials: surfactant, oil, gas}

A single surfactant is used, sodium dodecylsulfate (SDS), from Sigma Aldrich. Two different oils are used: $n$-dodecane (ACROS Organics, Geel, Belgium) and a commercial rapeseed oil (from Leaderprice, France) which is a mixture of triglycerides, with the presence of mono- and di-glycerides. MilliQ water is used in the preparation of all the samples. Due to the slow hydrolysis of SDS, the SDS aqueous solutions were prepared at most one day before use.

The two oils are chosen for their different interfacial tensions, which in turn translate to different entry, bridging and spreading coefficients. Although the antifoam activity is only indirectly linked to these coefficients, ${ }^{26}$ they are indicative of the antifoam potential of the oils. The entry coefficient $E$ is linked to the ability of the oil dispersed in water to penetrate into the air-water interface, it should be positive for the emulsion to act as an antifoam at all. While the bridging coefficient $B$ is linked to the ability of the oil globules to bridge the foam films, positive values indicate that the oil is potentially a fast antifoam. They are defined as follows:

$$
E=\gamma_{\mathrm{gw}}+\gamma_{\mathrm{ow}}-\gamma_{\mathrm{go}}, \quad B=\gamma_{\mathrm{gw}}^{2}+\gamma_{\mathrm{ow}}^{2}-\gamma_{\mathrm{go}}^{2}
$$

where the subscripts refer to the interfacial tension between gas $(\mathrm{g})$, water $(\mathrm{w})$ or oil (o) phases.

The interfacial tension measurements are either taken from literature $^{28,29}$ or measured using a drop (bubble) tensiometer (Tracker, Teclis, France). The interfacial tension between air and pure water is high, equal to $72 \mathrm{mN} \mathrm{m}$ m $^{-1}$ and without any surfactant; $E$ and $B$ are both positive for the two oils in our study. All the interfacial tension values are summarised in Table 1 for both the rapeseed oil and the dodecane, all values refer to an $8 \mathrm{~g}$ $\mathrm{L}^{-1} \mathrm{SDS}$ as the aqueous phase. In systems with dodecane, both $E$ and $B$ are still positive: $E_{\mathrm{dod} / \mathrm{SDS}}=18 \pm 3 \mathrm{mN} \mathrm{m}^{-1}$ and $B_{\mathrm{dod} / \mathrm{SDS}}=$ $740 \pm 150 \mathrm{mN}^{2} \mathrm{~m}^{-2}$, and the oil is still an antifoam. However, for rapeseed oil, $E$ becomes very small $E_{\mathrm{rs} / \mathrm{SDS}}=3 \pm 3 \mathrm{mN} \mathrm{m}^{-1}$, and $B$ is negative $B_{\mathrm{rs} / \mathrm{SDS}}=-57 \pm 150 \mathrm{mN}^{2} \mathrm{~m}^{-2}$, so this oil might not be an antifoam.

Two different gases have been used for the foams $-\mathrm{N}_{2}$ and $\mathrm{C}_{2} \mathrm{~F}_{6}$ - with different solubilities and diffusivities, in order to tune the ageing timescales of the system. It is known that foams made with $\mathrm{C}_{2} \mathrm{~F}_{6}$ have much slower coarsening rates than those from $\mathrm{N}_{2}$, and the slower coarsening will also have an impact of slowing down the drainage due to the coupling of these ageing processes. ${ }^{4}$

\subsection{Emulsion preparation}

Emulsions with varying volume fractions of oil are prepared $\left(\phi_{\text {oil }}=10,30,50\right.$ and $\left.70 \%\right)$ by mixing the aqueous phase in which the SDS has been dissolved $\left(0.5-16 \mathrm{~g} \mathrm{~L}^{-1}\right)$ with oil using an ultraturrax (IKAT 18 basic) at $20000 \mathrm{rpm}$ for 30 seconds. The size and polydispersity of the emulsion droplets is measured using a Malvern Mastersizer, Malvern Instruments, France. The preparation process results in rather polydisperse emulsions, with a polydispersity of around $50 \%$. The average emulsion drop radius also varies slightly with the concentration of emulsion decreasing from 5 to $2.5 \mu \mathrm{m}$ as the oil volume fraction increases to $70 \%$.

\subsection{Foamulsion generation}

The foamulsions are always prepared from the previously prepared emulsions, using the high pressure turbulent mixing method, ${ }^{30}$ where the gas and the emulsion are injected at high pressure into a T-junction module. This device has been developed for producing large volumes of foams of controlled and homogeneous liquid fractions $\varepsilon$ (typical production rate $6 \mathrm{~L}$ $\min ^{-1}$, with a liquid fraction $\varepsilon$ tuneable between 2 and $50 \%$ ).

In this study, the liquid pressure was kept constant at 5 bars for all experiments and the gas pressure was varied between 1.5 and 3 bars. For solutions that foam well, increasing the gas pressure leads to drier foams: in our case for solutions with good foamability, the foams have $\varepsilon \approx 5 \%$ with a gas pressure of 3 bars and $\varepsilon$ $\approx 10 \%$ with 1.5 bars. The full sets of data have been done with

Table 1 The interfacial tension values for both rapeseed oil and dodecane between gas, oil and an aqueous SDS solution with a concentration of $8 \mathrm{~g} \mathrm{~L}^{-1}$

\begin{tabular}{llll}
\hline $8 \mathrm{~g} \mathrm{~L}^{-1} \mathrm{SDS}$ & $\gamma_{\mathrm{gw}} / \mathrm{mN} \mathrm{m}^{-1}$ & $\gamma_{\mathrm{ow}} / \mathrm{mN} \mathrm{m}^{-1}$ & $\gamma_{\mathrm{go}} / \mathrm{mN} \mathrm{m}^{-1}$ \\
\hline Rapeseed & $37 \pm 1$ & $4 \pm 1$ & $36 \pm 1$ \\
Dodecane & $37 \pm 1$ & $7 \pm 1$ & $25 \pm 1$ \\
\hline
\end{tabular}




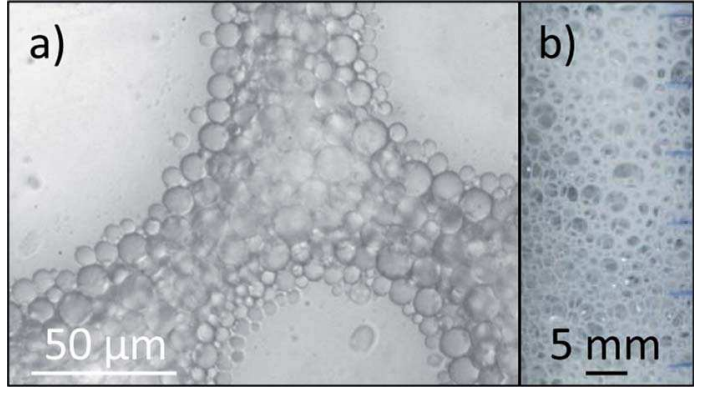

Fig. 1 Optical microscopy photograph of a rapeseed foamulsion with $\phi_{\text {oil }}=70 \%$ immediately after preparation (a) where the scale bar is $50 \mu \mathrm{m}$ and (b) a photograph 6 hours after preparation the scale bar is $5 \mathrm{~mm}$.

a gas pressure of 2 bars for the dodecane foamulsions and 3 bars for the rapeseed ones. The resulting mean bubble diameter (determined by optical microscopy) is always of $90 \pm 20 \mu \mathrm{m}$ with low polydispersity. Note that changing the gas has no impact on the foaming process. A photograph of a foamulsion is shown in Fig. 1(a) under the microscope straight after preparation and (b) in a photograph 6 hours after preparation, where the white Plateau borders filled with emulsion drops can be seen.

\subsection{A criterion for foamability}

Not all liquid solutions can produce foam of high quality, meaning a full incorporation, without loss, of the gas injected inside the fluid. For instance, concentrated surfactant solutions have generally high or maximal foamability, while pure liquids have low foamability. There is no absolute measure of the foamability of a solution, although many different methods exist for testing how much foam can be created from a given solution, such as the Ross-Miles test and bubbling methods. ${ }^{26}$ Indeed, it is very important to note that the foamability of the solution strongly depends on the foam generation process.

Here, we introduce a comparative foamability criterion, where the foamability is compared to that of a solution which is known to foam well. In Fig. 2 the initial liquid fraction, $\varepsilon$, of different

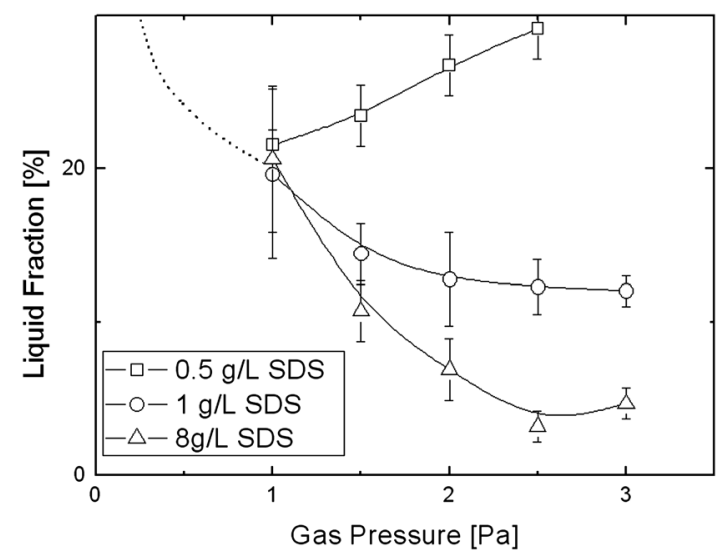

Fig. 2 Initial liquid fraction in SDS foams as a function of the $\mathrm{N}_{2}$ gas pressure in the turbulent mixing, the liquid pressure being kept constant at 5 bars. Three different concentrations of SDS are studied, $0.5 \mathrm{~g} \mathrm{~L}^{-1}$ (squares), $1 \mathrm{~g} \mathrm{~L}^{-1}$ (circles) and $8 \mathrm{~g} \mathrm{~L}^{-1}$ (triangles). The lines are guides for the eye. foams is shown as a function of gas pressure (liquid pressure was kept constant at 5 bars). With $8 \mathrm{~g} \mathrm{~L}^{-1} \mathrm{SDS}, \varepsilon$ decreases with increasing gas pressure, therefore the amount of air in the foamulsion increases. Solutions with $8 \mathrm{~g} \mathrm{~L}^{-1}$ are known to foam well, therefore these samples are taken as the reference. For our comparative scale, we choose to link the foamability of the solution to the initial liquid fraction of the produced foam: the higher the liquid fraction $\varepsilon$ obtained, the lower the foamability of the solution (less air trapped). This definition is meaningful only because here the bubble size remains roughly constant. In order to fully validate our reference, several different gas pressures and SDS concentrations are used for foamability tests. For low gas pressures, wet foams $(\varepsilon=20 \%)$ are obtained even for the highest SDS concentrations. However, as the gas pressure is increased the liquid fractions begin to depend on the SDS concentration. With $0.5 \mathrm{~g} \mathrm{~L}^{-1}$ SDS, $\varepsilon$ is always larger than $20 \%$, and the foamability even seems to decrease at higher pressures. Note that when the gas pressure tends to 0 , only liquid must be obtained ( $\varepsilon \rightarrow 100 \%$ ), so all the curves have to reach asymptotically the value $\varepsilon=100 \%$. This implies that at least with $0.5 \mathrm{~g} \mathrm{~L}^{-1} \mathrm{SDS}$ there is a minimum in the liquid fraction.

Doubling the SDS concentration to $1 \mathrm{~g} \mathrm{~L}^{-1}$ changes the behaviour considerably. With higher gas pressures $\varepsilon$ does decrease; however it reaches a minimum limit of around $12 \%$, whereas with higher concentrations of $\operatorname{SDS}\left(8 \mathrm{~g} \mathrm{~L}^{-1}\right) \varepsilon$ decreases down to around $5 \%$. From these data, we see that the foamability differences are better evidenced at the pressure of 2 bars and above, when the minimum value of $\varepsilon$ is reached for the concentrated surfactant solutions. We therefore defined $\varepsilon^{*}=20 \%$ as the limit between good and bad foamability for the two gas pressures used ( 2 and 3 bars).

\subsection{Monitoring foamulsion ageing}

The stability of the produced foamulsion is measured by imaging a foam column of initial height $h$ and by following the change in $h$. The time $t_{1 / 2}$ taken for $h$ to decrease by a factor 2 is a measure of the onset of coalescence and foam collapse, rather than ageing by drainage or coarsening (that produces small $h$ variations). ${ }^{31}$

The ageing - before collapse - of some of the most stable foamulsions was followed by measuring the evolution of $\varepsilon$ by conductivity, with pairs of electrodes incrusted in the cell walls, and using the calibration curves provided in. ${ }^{32}$ In the measurements, the conductivity of the emulsion is considered constant and is used as reference. We will come back to this point when analysing these data.

\section{Results and discussion}

\subsection{Conditions for the production of the foamulsion: foamability of the emulsion}

We have compared the effects of changing the SDS concentration, $c_{\mathrm{SDS}}$ and the volume fraction of oil in the emulsion, $\phi_{\mathrm{oil}}$. The variation of the initial liquid fraction in the samples as a function of SDS concentration is shown in Fig. 3 for the SDS - dodecane foamulsion made using a gas pressure of 2 bars. At high $c_{\mathrm{SDS}}$, the initial water content of the foam is around $9 \pm 2 \%$, with or without oil, and as $c_{\text {SDS }}$ decreases $\varepsilon$ increases in a continuous manner. The criterion for good foamability, $\varepsilon<\varepsilon^{*}=20 \%$, allows 


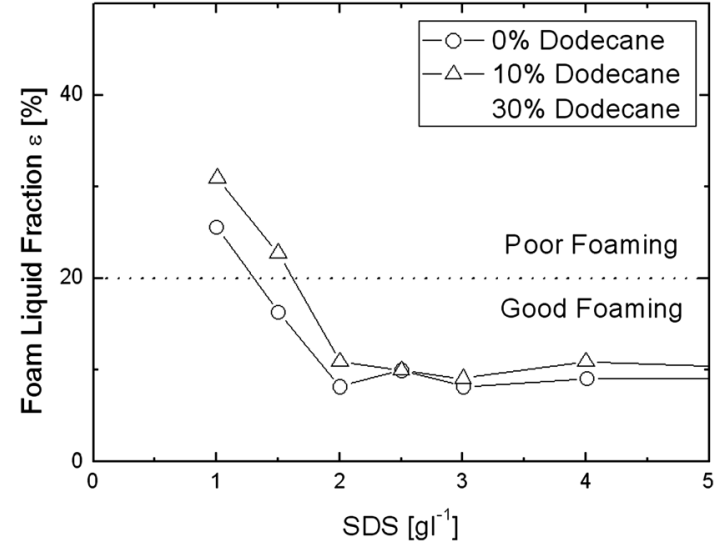

Fig. 3 Initial liquid fraction $\varepsilon$ in the foam as a function of SDS concentration for three different dodecane volume fractions in the emulsion $\phi_{\text {oil }}(0,10$ and $30 \%)$ for a gas pressure of 2 bars. The dashed line indicates the limit of good foamability, $\varepsilon^{*}=20 \%$.

us to classify all the samples into good and poor foamers and to build up a "phase diagram" of foamability as a function of $c_{\mathrm{SDS}}$ and $\phi_{\text {oil }}$. Choosing a value slightly different for $\varepsilon^{*}$ does not induce strong changes in the phase diagram.

Such phase diagrams for rapeseed oil and dodecane are shown in Fig. 4 and 5, respectively. The two diagrams are qualitatively identical, with two regimes separated by a critical concentration of SDS, $c^{*}$ sDs. This critical concentration increases with increasing oil content in the samples (starting from around $1 \mathrm{~g}$ $\mathrm{L}^{-1}$ when no oil is added).

A third region, above $c^{*}$ SDs, and at high $\phi_{\text {oil }}$ is found, where the liquid fraction in the foams is above $20 \%$ after preparation (triangles Fig. 3), but the foams are very stable. The photo of the foam 6 hours after preparation is shown in Fig. 1b. Here the emulsion is very concentrated and viscoelastic, as we are above the random close packing of spheres. The poor foamability could be due to the high viscosity of the continuous phase, and experiments with concentrated solutions of glycerol confirmed such an effect.

Let us attempt to rationalise the variation of $c^{*}$ SDS $v s$. $\phi_{\text {oil }}$. Addition of oil can have two effects: a dilution effect, the concentration of SDS in the emulsion being actually smaller than in water by a factor $\left(1-\phi_{\mathrm{oil}}\right)$, and adsorption of SDS at the interfaces of the oil droplets, which also lowers the effective SDS concentration. As the area covered by one SDS molecule $T$ is $50 \AA^{2}$ and the molecular weight of SDS is $M_{\mathrm{w}} 288.36 \mathrm{~g} \mathrm{~mol}^{-1}$, we can calculate the concentration of SDS at the surface of the emulsion droplets as:

$$
c_{\text {drop }}=\frac{3}{R_{\text {drop }}} \phi_{\text {oil }} \frac{M_{\mathrm{w}}}{N_{\mathrm{A}} \Gamma}=3 \alpha \phi_{\text {oil }}
$$

where $R_{\text {drop }}$ is the radius of emulsion drops, varying from 2.5 to $5 \mu \mathrm{m}$. Combining this with the effect of dilution results in an effective concentration of free SDS, $c_{\text {eff }}$, in the emulsion:

$$
c_{\text {eff }}=\left(1-\phi_{\text {oil }}\right) c_{\mathrm{SDS}}-\frac{3 \phi_{\text {oil }} M_{\mathrm{w}}}{R_{\mathrm{drop}} \Gamma N_{\mathrm{A}}}
$$

From eqn (3), one finds that the adsorption effect becomes significant only after $\phi_{\text {oil }}=40 \%$ for the $R_{\text {drop }}$ used in this study.

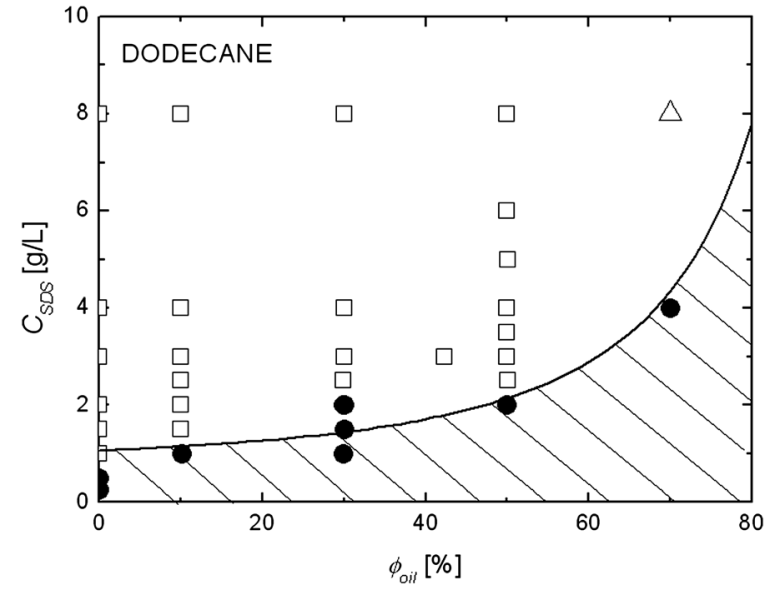

Fig. 4 Liquid fraction, $\varepsilon$, as a function of SDS concentration, $c_{\mathrm{sds}}$, and oil volume fraction, $\phi_{\text {oil }}$, within the emulsion for the dodecane system and a gas pressure of 2 bars. The filled circles indicate samples that do not foam well, the empty black squares those that foamed well, and the empty triangles samples that had a large initial $\varepsilon$, but were very stable in time. The solid line is the best fit using eqn (4).

At $\phi_{\text {oil }}=0$, the criterion for good foamability can be expressed in terms of a critical free SDS concentration in the solution; $c_{\mathrm{SDS}}=$ $c_{\text {eff }}>c_{0}$. As eqn (3) gives the effective free SDS concentration in the emulsion to be foamed, a possible criterion of good foamability could be that the concentration of free SDS must be higher than a constant critical concentration, $c_{0}$, independent of the fluid (and then of $\phi_{\mathrm{oil}}$ ). Assuming such a relation, it follows that:

$$
c_{\mathrm{SDS}}^{*}=\frac{c_{0}+3 \alpha \phi_{\mathrm{oil}}}{1-\phi_{\mathrm{oil}}}
$$

The best fit to the data with eqn (4) is shown in Fig. 4 for the dodecane system using $c_{0}=1.1 \mathrm{~g} \mathrm{~L}^{-1}$. One sees that a good agreement is found: the line in Fig. 4 follows well the frontier between the two regimes. This proves that the criterion of good foamability is simply a critical effective concentration of SDS, whatever the amount of oil in the emulsion. This agreement also implies that the SDS molecules captured at the droplets'

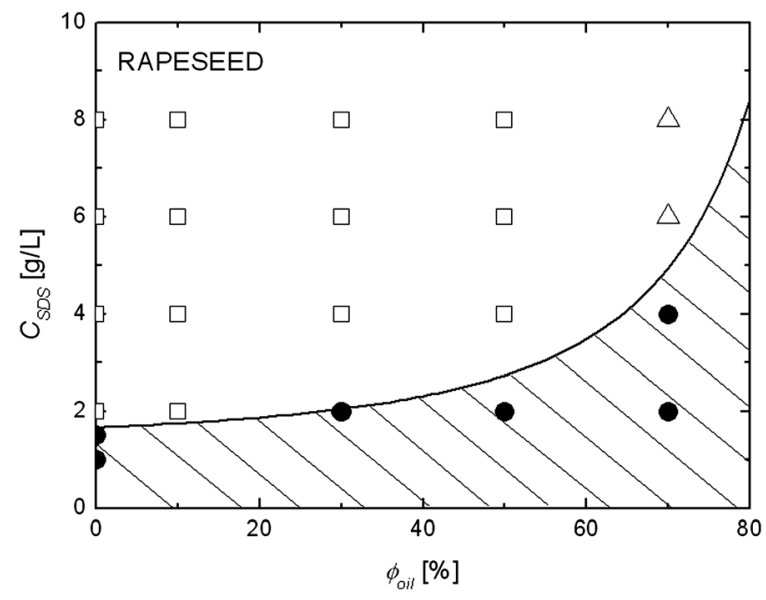

Fig. 5 Same foamability diagram as in Fig. 4; the rapeseed oil system with a gas pressure of 3 bars. The solid line is the best fit using eqn (4). 
interfaces do not help to foam. This is especially clear in the range $\phi_{\text {oil }}>40 \%$, where the adsorption effect is no longer negligible and a proper calculation of the stability limit require taking it into account. Therefore, the surfactant initially on the emulsion droplets remains there, and even the vigorous turbulent mixing during the generation of the foam is unable to change the surfactant partitioning between the oil drops and the air bubbles. This means that the reason why the oil suppresses foaming at low SDS concentrations is that it both dilutes the initial solution and captures surfactant at the oil drop surfaces.

The model also works well for the foamulsion made with rapeseed oil, and the stability limit is well described (Fig. 5). We find a slightly different $c_{0}, 1.7 \mathrm{~g} \mathrm{~L}^{-1}$, when with dodecane it was $1.1 \mathrm{~g} \mathrm{~L}^{-1}$; this means that the foamability limit (even with $\phi_{\text {oil }}=$ 0 ) is different for the two sets of data. In fact this difference seems due to the fact that the dodecane foamulsions were prepared using a gas pressure of 2 bars, whereas the rapeseed foamulsions were prepared using a higher gas pressure, 3 bars. This is further corroborated by a third phase diagram (not shown) prepared with rapeseed oil using a gas pressure of 1.5 bars, where $c_{0}$ is even lower at $0.9 \mathrm{~g} \mathrm{~L}^{-1}$. Therefore, the differences in $c_{0}$, although difficult to rationalise, seem less due to the type of oil than to the preparation of the foam.

\subsection{Foamulsion evolution in time}

Let us now focus on the systems of high foamability, corresponding to the top regions of the phase diagrams of Fig. 4 and 5. From simple visual observations, just after the production, the foamulsions are homogeneously white samples with uniform bubble sizes. With time, these foams age, and eventually collapse. The variation of foam height with time for the different samples changes considerably with $\phi_{\text {oil }}$, as illustrated in Fig. 6 for the solutions containing $8 \mathrm{~g} \mathrm{~L}^{-1} \mathrm{SDS}$ and dodecane. The sample with $\phi_{\text {oil }}=10 \%$ starts to collapse after 20 minutes, while those with $\phi_{\text {oil }}=30$ or $50 \%$ start to collapse almost immediately. It is only with $\phi_{\text {oil }}=70 \%$ that the foamulsion becomes more stable again. The foamulsion lifetime $t_{1 / 2}$ (time to reach half of the initial height) is plotted as a function of $c_{\mathrm{SDS}}$ and $\phi_{\text {oil }}$ for the two oils in

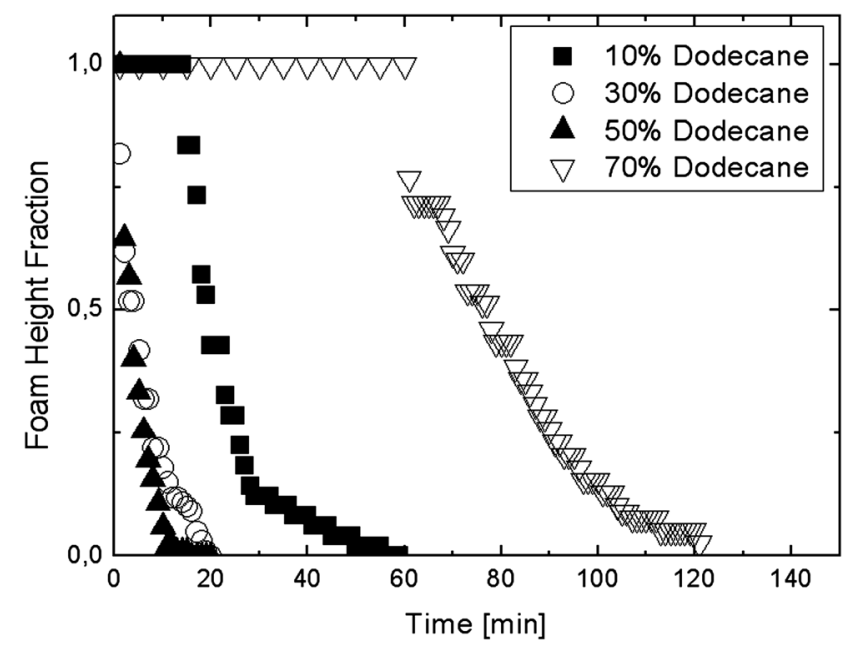

Fig. 6 Foamulsion height as a function of time for an $8 \mathrm{~g} \mathrm{~L}^{-1}$ SDS solution with different volume fractions of dodecane $(10-70 \%)$.
Fig. 7. Note that the foam column top boundary is easy to determine with the foamulsions, as the liquid is itself milky; so the largest error bars are found for $\phi_{\text {oil }}=0$. Significantly different results are found depending on the oil used.

For rapeseed oil (with $\phi_{\text {oil }}<70 \%$ ), the foams have lifetimes that are very much comparable with those of the samples prepared with SDS only and the addition of oil has very little influence on the foam stability (Fig. 7a). The only effect is seen at high $\phi_{\text {oil }}$, where the lifetime of the foamulsion drastically increases, reaching lifetimes at least ten times higher than for $\phi_{\text {oil }}=0$.

In contrast, the presence of dodecane in the emulsion decreases the lifetime strongly (Fig. 7b). Up to $\phi_{\text {oil }}=50 \%$ the foam collapses within minutes of preparation. Even large amounts of SDS are not sufficient to recover oil-free foam lifetimes and only

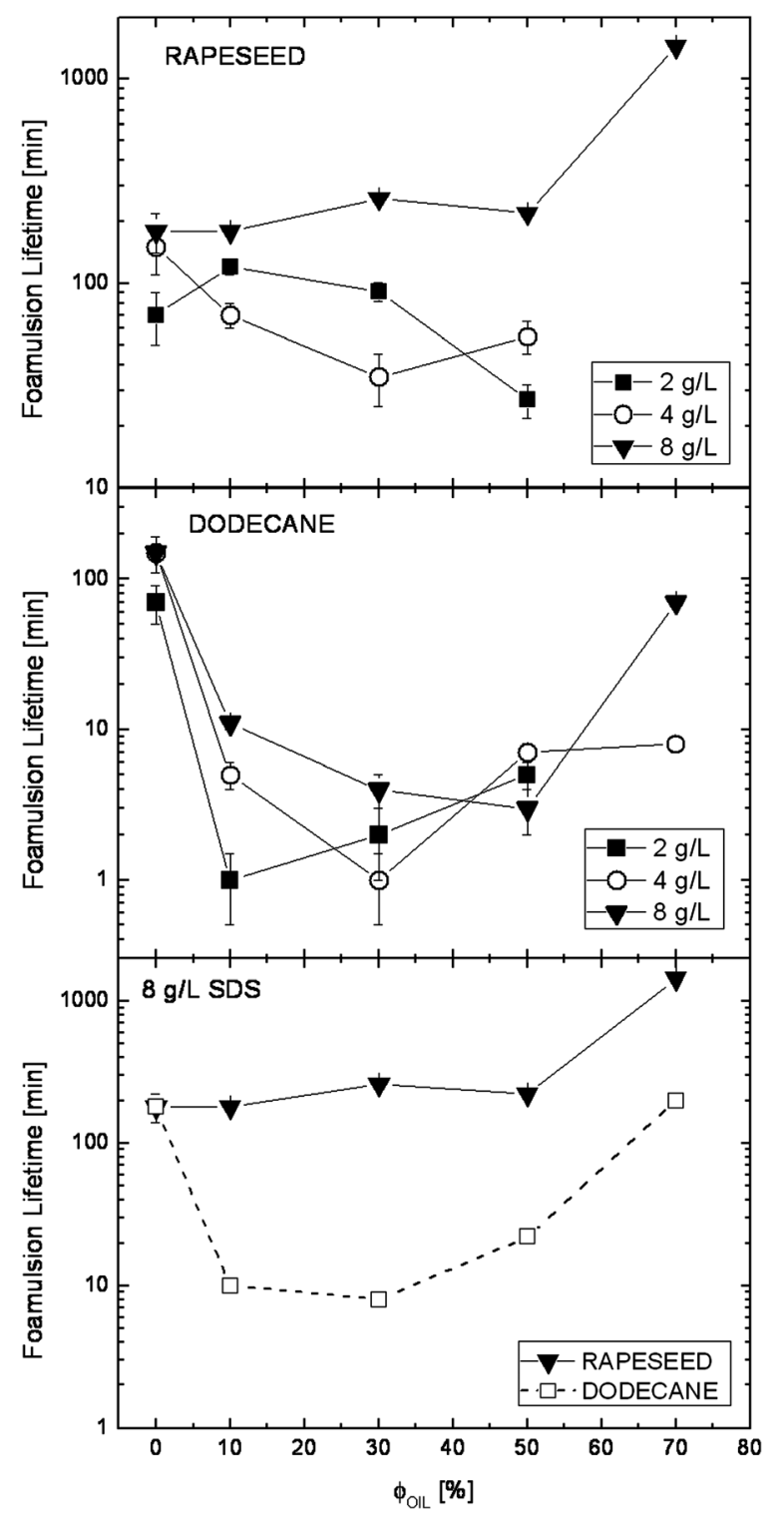

Fig. 7 Foamulsion lifetime as a function of oil content in the emulsion, for SDS concentrations in $\mathrm{g} \mathrm{L}^{-1}: 2$ (squares), 4 (circles), 8 (triangles down) for (a) rapeseed oil and (b) dodecane. The curves with $8 \mathrm{~g} \mathrm{~L}^{-1} \mathrm{SDS}$ for both oils are repeated in (c) to facilitate comparison and the much longer foam lifetimes of rapeseed oil foamulsions are clearly observed. 
at high $\phi_{\text {oil }}(70 \%)$ does the foamulsion life time return to the levels found without added dodecane (provided $c_{\mathrm{SDS}}>c_{0}$ ). In Fig. 7c the lifetimes for foamulsions prepared with $8 \mathrm{~g} \mathrm{~L}^{-1} \mathrm{SDS}$ for both rapeseed oil and dodecane are shown, where the difference in the stabilities is consistently almost an order of magnitude in favour of the rapeseed oil foamulsions.

Let us first discuss the difference in the intermediate regime, $10 \%<\phi_{\text {oil }}<60 \%$. For the rapeseed oil, the foamulsions drain and coarsen as classical aqueous foams made with SDS. Once the bubbles are large ( $\mathrm{mm}$ in diameter) and the Plateau borders are highly shrunk, the films between bubbles break and the structure collapses. This suggests that the oil droplets are only transported through the water, and they do not interfere with the gas-liquid interfaces, which is consistent with the very small value of entry coefficient $E$.

In contrast for dodecane, for which both $E$ and $B$ are negative, the foamulsions are much less stable. The differences between the two oils seem in this case rather well discriminated by the values of $E$ and $B$. Not only the dodecane systems have a lower lifetime than oil-free foams, but collapse occurs before drainage is complete. Thus, the choice of oil appears to have a much stronger impact on foam stability, than on the foamability. This is linked to the different timescales of the processes: in the stability experiments, the foam ages and oil droplets have time to act, whereas the foaming process is too fast for this to occur. This of course implies that dodecane acts as slow antifoam; the oil droplets do not enter the films, but break the foam only after being squeezed inside the Plateau borders.

Indeed the destabilising influence of dodecane is clear as the samples are studied under the microscope. For intermediate dodecane concentrations $\left(30 \%<\phi_{\text {oil }} 50 \%\right)$ it was not possible to image the foamulsion at all due to its limited stability (simply placing the foam between microscope slides breaks it immediately). However at $\phi_{\text {oil }}=70 \%$ the foamulsion becomes sufficiently stable to be observed under the microscope as shown in Fig. 8a, where densely packed droplets are seen around the bubbles.

The foamulsions prepared from rapeseed oil can be more easily manipulated as pictured straight after preparation in Fig. $8 \mathrm{~b}\left(\phi_{\text {oil }}=30 \%\right)$, where it can be seen to resemble closely a foam doped with particles. Some of the Plateau borders have thinned into the junctions, where the droplets are highly packed. Indeed observing such foams we can see that there are thin films formed between the bubbles.

With $\phi_{\text {oil }}=70 \%$, very different features are observed, in particular an outstandingly long lifetime with rapeseed oil. A first important point for understanding this new regime is that as $\phi_{\text {oil }}$ $>63 \%$ (random close packing of spheres), the emulsion droplets are highly packed, ${ }^{33}$ and the emulsion becomes viscoelastic, with finite shear modulus and yield stress. Microscope images of such a foamulsion $\left(\phi_{\text {oil }}=70 \%\right)$ are shown in Fig. $8 \mathrm{c}$ and d (rapeseed oil). One can see that droplets are actually confined and crowded between bubbles, which stay anomalously far from each other. The presence of such a dense assembly of droplets trapped and jammed in between the bubbles has several effects. The local viscosity increases, and both film thinning and Plateau borders shrinking are slowed down (slower drainage). In addition, for initial bubble diameters of order $100 \mu \mathrm{m}$, hydrodynamic stresses in the Plateau borders become comparable to the yield stress of the emulsion (of the order of a few $\mathrm{Pa}^{34,35}$ ). Drainage can

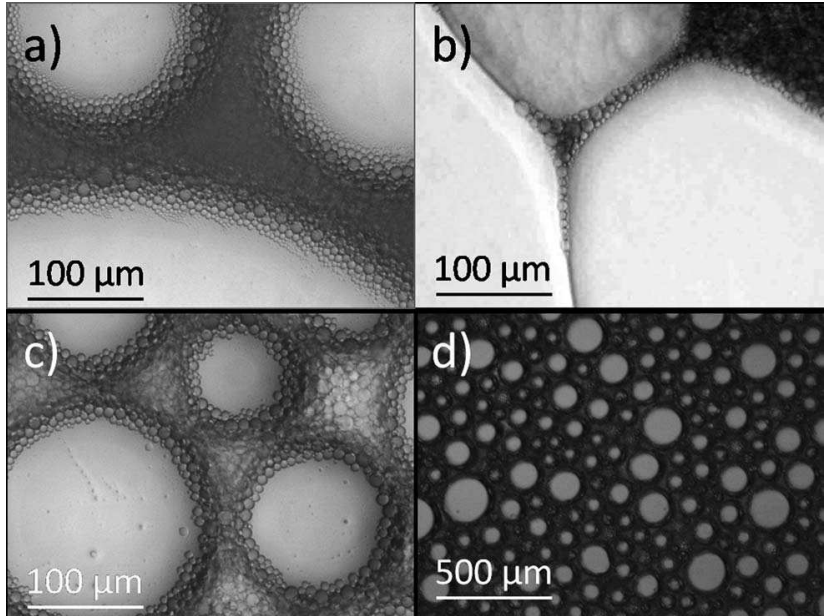

Fig. 8 Microscopy images of foamulsions straight after preparation: (a) from dodecane with $\phi_{\text {oil }}=70 \%$, (b) rapeseed oil with $\phi_{\text {oil }}=30 \%$, (c) and (d) rapeseed oil with $\phi_{\mathrm{oil}}=70 \%$ (different magnifications). In all the cases the emulsion droplets have a size of around $10 \mu \mathrm{m}$, and in (a) and (b) the bubble size has slightly increased (from the initial $100 \mu \mathrm{m}$ ) due to coalescence as the foamulsions are placed under the cover slips: the droplets have been chased out into the Plateau borders from the thin films.

therefore not only be slowed down, but it can even be arrested if the yield stress of the emulsion becomes higher than the local hydrodynamic stresses. ${ }^{13}$ Moreover, in the pictures, one can also see that the droplets assemble at the interfaces, packing like solid particles. As for solid-stabilized bubbles, this armour of droplets at the interface might result in a slowing down of the coarsening. ${ }^{9}$ In any case, the coarsening rate is surely reduced by the large thickness of the films. Therefore, in these systems both drainage and coarsening are strongly slowed down. As these two effects are coupled in such a synergetic way that they enhance each other, ${ }^{4}$ slowing down both of them a little leads to a much slower global ageing, and thus much longer lifetimes. Therefore, the use of concentrated emulsions for foaming leads to very good stability through the combination of bulk viscoelastic effects, drastically decreasing the drainage velocity, and the presence of thick layers of droplets covering the bubbles, strongly decreasing gas exchange and coarsening rates. Nevertheless, as often encountered for foams, the drawback of using such concentrated emulsions is that-even though the resulting foamulsions have excellent stability — their foamability becomes poor.

\subsection{Further optimisation: gas effect}

In order to modify the relative timescales of the ageing processes, we performed additional measurements with a different gas, replacing $\mathrm{N}_{2}$ by $\mathrm{C}_{2} \mathrm{~F}_{6}$. The lifetime of a foam made of $\mathrm{C}_{2} \mathrm{~F}_{6}$, compared to $\mathrm{N}_{2}$, is much longer because both drainage and coarsening timescales are increased due to the coupling of the two. ${ }^{4}$

Fig. 9 shows typical drainage curves (i.e. local fluid fraction as a function of time at a given vertical position) of a $\mathrm{C}_{2} \mathrm{~F}_{6}$ foamulsion with an initial $\phi_{\text {oil }}=30 \%$ of rapeseed oil. One can see that, for typical ageing times of 200 minutes, already corresponding to the full lifetime with $\mathrm{N}_{2}$ (Fig. 7a), the local fluid fraction has roughly halved, but no collapse has occurred. So, the stability of a $30 \%$ oil foamulsion is strongly increased with $\mathrm{C}_{2} \mathrm{~F}_{6}$, 


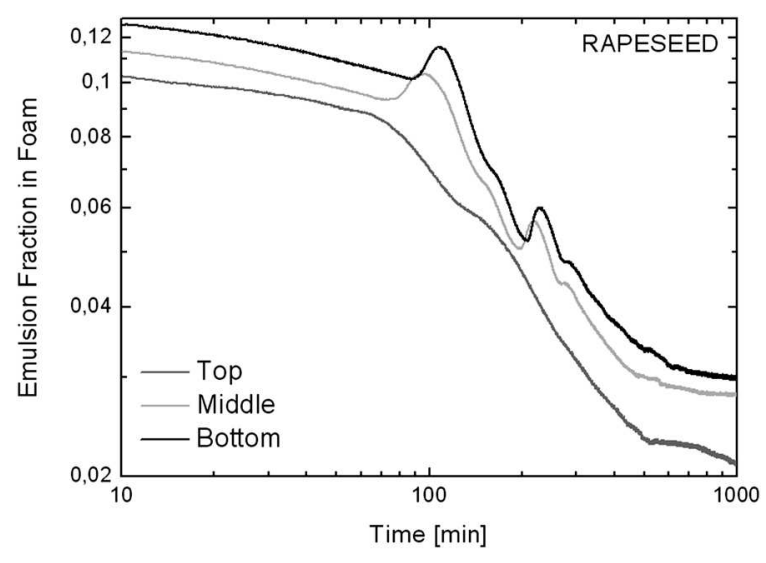

Fig. 9 Time evolution of the local fluid fraction in a foamulsion, prepared with $\mathrm{C}_{2} \mathrm{~F}_{6}$, and made of an emulsion containing $30 \%$ of rapeseed oil. The measurements are made at 3 vertical positions. The anomalous decrease of $\varepsilon$, with peaks, indicates a stopping and restarting flow, which results in propagating fluid pulses.

and becomes comparable to what is found at higher $\phi_{\text {oil }}$ with $\mathrm{N}_{2}$. In comparison foams with SDS at $8 \mathrm{~g} \mathrm{~L}^{-1}$ made with $\mathrm{C}_{2} \mathrm{~F}_{6}$ still drain rather rapidly (although more slowly than with $\mathrm{N}_{2}$ ), where the liquid fraction is below 0.01 in 10 s of minutes.

The effect can be further understood by studying the shape of the draining curves in Fig. 9. Once the liquid fraction starts to decrease, the rate is not monotonous and peaks in conductivity are observed at different heights. These peaks correspond to local excesses of liquid, which propagate downward, like pulses or avalanches (at typically $3 \mathrm{mms}^{-1}$ ). Such features have been observed previously only with confined viscoelastic fluids in the Plateau borders, ${ }^{13}$ and they are the signature of a stop-start of drainage, with jamming-unjamming of the flow. This is the result of the competition between gravity-induced shrinking of the Plateau border sections (leading to slowed down or arrested drainage) and coarsening-induced deconfinement (re-opening the Plateau borders to restart the flow). In this case, despite an initial oil fraction of the emulsion $\phi_{\mathrm{oil}}=30 \%$ (i.e. fluid-like emulsion), the continuous phase flowing through the foamulsion has become viscoelastic: the emulsion has actually aged by creaming within the Plateau borders. The ageing of the foam structure has been reduced so much by the use of $\mathrm{C}_{2} \mathrm{~F}_{6}$ that the fastest ageing process is the creaming of the emulsion inside the Plateau borders (with $\phi_{\text {oil }}=30 \%$ and $R_{\text {drop }}=5 \mu \mathrm{m}$, creaming takes only minutes). In these conditions, since the fluid in the Plateau borders has changed, the conductivity measurements have to be taken with caution: the reference is actually changing with time. Therefore, a full quantitative analysis is not possible, but the qualitative features discussed above remain valid.

Two different routes have been identified for the production of very stable foamulsion: either foaming an already concentrated emulsion or a dilute emulsion, which ages towards a concentrated emulsion faster than the foam Plateau border network evolution, in which it is confined.

\section{Summary and conclusions}

We were able to produce large volumes of well-controlled dual oil/gas in water dispersions, through a foaming process of emulsions (introducing the name foamulsion). It turns out that such foamulsions show a large variety of behaviours. Playing with the different ingredients and their proportions-oil, surfactant, gas - we have identified several regimes: a regime where no foam is obtained, a regime where foam is produced but has a very low stability and rapidly collapses, a regime where the foam lifetime is identical to the oil-free foams and a regime of enhanced stability (lifetime is strongly increased by the presence of the oil droplets).

Regarding the critical conditions required for producing these foamulsion, we have been able to explain the existence of a critical surfactant concentration by two uncoupled effects: surfactant dilution by the oil and surfactant adsorption on the oil droplets. These results show that once the surfactant is adsorbed onto the oil droplets it is captured and does not leave these interfaces to stabilize newly created gas-liquid interfaces: only the remaining free surfactants are available to stabilize the foam. In that respect, the ability to disperse gas into the emulsion is found to be independent of the type of oil. However, the stability of the foamulsions is very different, and dodecane although inactive during foaming (provided it is fast enough), afterwards breaks the foams within minutes.

We have clearly identified a very stable regime, obtained when large oil fractions are used. Microscopic pictures and emulsion properties at high oil fraction help to understand the origin of this enhanced stability. It arises from an original combination of bulk viscoelasticity (jamming of the droplets) and yielding on one side and of thick layers of droplets around the bubbles on the other side. Note that if the oil fraction is too high, the stability may rise only if there is enough free surfactant present in water to compensate for adsorption and dilution effects.

The results also show that macroscopic features are widely controlled by the competition between various timescales, all being controlled by the type and amount of oil, sizes of the droplets and bubbles, type of gas, and type and amount of surfactant. Here, we have identified the ones associated to: the production device, the antifoaming activity of oil droplets, the drainage of the emulsion through the Plateau borders network, the gas transfer between bubbles, and the ageing of the emulsion. The tests done with $\mathrm{C}_{2} \mathrm{~F}_{6}$ illustrate well the interplay between foam and emulsion aging, emulsion creaming in this case being faster than foam drainage.

Let us finally stress that simply by mixing water, oil, gas and surfactant, one obtains foamulsions with lifetimes ranging from one to thousands of minutes. In these systems, the stabilisation regimes are all very different, and their understanding is important for practical and industrial applications.

\section{Acknowledgements}

The authors thank the ANR for funding through the ANRBLAN-07-340 and Reine-Marie Guillermic for stimulating discussions on drainage with viscoelastic fluids.

\section{References}

1 D. Weaire and S. Hutzler, The Physics of Foams, Oxford, Oxford University Press, 1999.

2 B. P. Binks, Modern Aspects of Emulsion Science, The Royal Society of chemistry, 1998. 
3 D. Langevin, Aqueous foams: a field of investigation at the frontier between chemistry and physics, ChemPhysChem, 2008, 9(4), 510-522.

4 A. Saint-Jalmes, Physical chemistry in foam drainage and coarsening, Soft Matter, 2006, 2, 836-849.

5 I. Cantat, et al., Les Mousses: Structure et Dynamique. Echelles, Belin, 2011.

6 S. Tcholakova, N. D. Denkov and A. Lips, Comparison of solid particles, globular proteins and surfactants as emulsifiers, Phys. Chem. Chem. Phys., 2008, 10(12), 1608-1627.

7 A. Saint-Jalmes, et al., Differences between protein and surfactant foams: microscopic properties, stability and coarsening, Colloids Surf., A, 2005, 263(1-3), 219-225.

8 B. P. Binks and T. S. Horozov, Aqueous foams stabilized solely by silica nanoparticles, Angew. Chem., Int. Ed., 2005, 44(24), 3722-3725.

9 A. C. Martinez, et al., On the origin of the remarkable stability of aqueous foams stabilised by nanoparticles: link with microscopic surface properties, Soft Matter, 2008, 4(7), 1531-1535.

10 R. G. Alargova, et al., Foam superstabilization by polymer microrods, Langmuir, 2004, 20(24), 10371-10374.

11 S. U. Pickering, CXCVI-Emulsions, J. Chem. Soc. Trans., 1907, 91, 2001-2021.

$12 \mathrm{~W}$. Ramsden, Separation of solids in the surface-layers of solutions and 'suspensions' (observations on surface-membranes, bubbles, emulsions, and mechanical coagulation)-preliminary account, Proc. R. Soc. London, 1903, 72(477-486), 156-164.

13 R. M. Guillermic, et al., Surfactant foams doped with laponite: unusual behaviors induced by aging and confinement, Soft Matter, 2009, 5(24), 4975-4982.

14 F. Carn, et al., Foam drainage in the presence of Nanoparticlesurfactant mixtures, Langmuir, 2009, 25(14), 7847-7856.

$15 \mathrm{~S}$. Cohen-Addad, et al., Rigidity percolation in particle-laden foams, Phys. Rev. Lett., 2007, 99(16), 168001-1-168001-4.

16 A.-L. Fameau, et al., Smart foams: switching reversibly between ultrastable and unstable foams, Angew. Chem., 2011, 123(36), 84148419

17 D. Varade, et al., On the origin of the stability of foams made from catanionic surfactant mixtures, Soft Matter, 2011.

18 A. S. Utada, et al., Monodisperse double emulsions generated from a microcapillary device, Science, 2005, 308(5721), 537-541.

19 G. Muschiolik, Multiple emulsions for food use, Curr. Opin. Colloid Interface Sci., 2007, 12(4-5), 213-220.
20 A. T. Florence, T. K. Law and T. L. Whateley, Nonaqueous foam structures from osmotically swollen w/o/w emulsion droplets, J. Colloid Interface Sci., 1985, 107(2), 584-588.

21 F. Leal-Calderon, F. Thivilliers and V. Schmitt, Structured emulsions, Curr. Opin. Colloid Interface Sci., 2007, 12(4-5), 206-212.

22 K. E. Allen, B. S. Murray and E. Dickinson, Development of a model whipped cream: effects of emulsion droplet liquid/solid character and added hydrocolloid, Food Hydrocolloids, 2008, 22(4), 690-699.

23 K. E. Allen, B. S. Murray and E. Dickinson, Whipped cream-like textured systems based on acidified caseinate-stabilized oil-in-water emulsions, Int. Dairy J., 2008, 18(10-11), 1011-1021.

$24 \mathrm{~K}$. Koczo, L. A. Lobo and D. T. Wasan, Effect of oil on foam stability-aqueous foams stabilized by emulsions, J. Colloid Interface Sci., 1992, 150(2), 492-506.

25 A. Salonen, D. Langevin and P. Perrin, Light and temperature biresponsive emulsion foams, Soft Matter, 2010, 6(21), 5308-5311.

26 N. D. Denkov, Mechanisms of foam destruction by oil-based antifoams, Langmuir, 2004, 20(22), 9463-9505.

27 C. A. Miller, Antifoaming in aqueous foams, Curr. Opin. Colloid Interface Sci., 2008, 13(3), 177-182.

28 CRC Handbook of Chemistry and Physics, Internet Version 2005, ed. David R. Lide, CRC Press, Boca Raton, FL, 2005, http://www. hpcbnetbase.com.

29 A. Bonfillon and D. Langevin, Viscoelasticity of monolayers at oilwater interfaces, Langmuir, 1993, 9(8), 2172-2177.

30 A. Saint-Jalmes, M. U. Vera and D. J. Durian, Uniform foam production by turbulent mixing: new results on free drainage $v s$. liquid content, Eur. Phys. J. B, 1999, 12(1), 67-73.

31 D. Georgieva, A. Cagna and D. Langevin, Link between surface elasticity and foam stability, Soft Matter, 2009, 5(10), $2063-$ 2071.

$32 \mathrm{~K}$. Feitosa, et al., Electrical conductivity of dispersions: from dry foams to dilute suspensions, J. Phys.: Condens. Matter, 2005, 17 (41), 6301-6305.

33 T. G. Mason, J. Bibette and D. A. Weitz, Yielding and flow of monodisperse emulsions, J. Colloid Interface Sci., 1996, 179(2), 439-448.

$34 \mathrm{R}$. Pal, Effect of droplet size on the rheology of emulsions, AIChE J., 1996, 42(11), 3181-3190.

35 R. Pal, Yield stress and viscoelastic properties of high internal phase ratio emulsions, Colloid Polym. Sci., 1999, 277(6), 583-588. 